D. Motreanu

Nagoya Math. J.

Vol. 106 (1987), 29-47

\title{
TANGENT VECTORS TO SETS IN THE THEORY OF GEODESICS
}

\author{
DUMITRU MOTREANU
}

\section{Introduction}

In the setting of Banach manifolds the notion of tangent vector to an arbitrary closed subset has been introduced in [11] by the author and N. H. Pavel, and it has been used in flow-invariance and optimization ([11], [12], [13]). For detailed informations on tangent vectors to closed sets (including historical comments) we refer to the recent book of N. H. Pavel [17].

The aim of this paper is to apply this general concept of tangency in the study of geodesics. We are concerned with geodesics which have either the endpoints in given closed subsets or the same angle for a fixed closed subset. This approach allows one to extend important results due to K. Grove [4] and T. Kurogi ([6], [7]).

Section 1 is devoted to the general theory of tangent vectors. Here we prove some useful results in critical point theory and existence for minimization.

In Section 2 we deal with geodesics as critical points of the energy functional relative to certain sets of paths. In this way we obtain characterizations for various geodesics between closed subsets. In addition, we point out that the energy functional satisfies a generalized version of Condition (C) relative to some sets (not necessarily submanifolds).

Section 3 contains the extension of the Kurogi's work on geodesics with the same angle. More precisely, we provide sufficient conditions insuring the existence of geodesics invariant with respect to an isometry and acrossing with the same angle a compact subset.

Acknowledgement. Part of this work has been presented in a Conference at the University of Freiburg on August, 1984. The author is very indebted to Professor M. Barner for kind support.

Received July 5, 1985. 


\section{§1. Tangent vectors and existence for minization}

This section contains a theory of tangency for arbitrary subsets of a

$C^{1}$ Banach manifold emphasizing its consequences in abstract minimization problems.

Let $M$ be a $C^{1}$ manifold modeled on a Banach space $E$ with the norm \|| $\|$ and let $S$ be a nonempty subset of $M$.

Definition 1.1 ([11]). A vector $v \in T_{x} M$ is called tangent to the subset $S$ of $M$ at $x \in S$ if there is a chart $\varphi: U \rightarrow E$ of $M$ at $x$ such that

$$
\lim _{h \rightarrow 0} \frac{1}{h} d\left(\varphi(x)+h D \varphi_{x}(v) ; \varphi(U \cap S)\right)=0
$$

(with $h \in R$ ).

Here $D \varphi_{x}: T_{x} M \rightarrow E$ is the differential of $\varphi$ at $x$ and $d(u ; A)$ denotes the distance in $E$ from a point $u \in E$ to a subset $A$ of $E$, i.e.

$$
d(u ; A)=\inf _{a \in A}\|u-a\| .
$$

Remark. Definition 1.1 is independent of the chart $(U, \varphi)$, that is if the vector $v \in T_{x} M$ is tangent to the set $S$ at $x \in S$ and $(V, \psi)$ is an arbitrary chart of $M$ at $x$, then

$$
\lim _{h \rightarrow 0} \frac{1}{h} d\left(\psi(x)+h D \psi_{x}(v) ; \psi(V \cap S)\right)=0 .
$$

The set of tangent vectors to a subset $S$ at $x \in S$ is always nonempty containing at least $0 \in T_{x} M$. In [11], [12] and [13] the tangent vectors describec. in Definition 1.1 are called quasi-tangent vectors.

A vector field $X: M \rightarrow T M$ is said to be tangent to a subset $S$ of $M$ if for each $x \in S$ the vector $X(x) \in T_{x} M$ is tangent to $S$ at $x$. According to the above Remark the vector field $X$ on $M$ is tangent to the set $S$ if and only if for every chart $(U, \varphi)$ of $M$ intersecting $S$ and every $x \in U \cap S$ we have

$$
\lim _{h \rightarrow 0} \frac{1}{h} d\left(\varphi(x)+h X_{\varphi}(x) ; \varphi(U \cap S)\right)=0,
$$

where $X_{\varphi}(x)=D \varphi_{x}(X(x))$ represents the principal part of the vector field $X$ in the chart $\varphi$.

In the case of submanifolds the tangency introduced in Definition 1.1 reduces to ordinary tangency. 
Proposition 1.1 ([11]). Let $S$ be a $C^{1}$ submanifold of $M$. A vector $v \in T_{x} M$ is tangent to $S$ at $x \in S$ in the sense of Definition 1.1 if and only if $v$ is a tangent vector to $S$ at $x$, i.e. $v \in T_{x} S$.

The following result shows the functorial character of the tangent vectors to sets.

Theorem 1.2 ([11], [13]). Let $f: M \rightarrow N$ be a $C^{1}$ mapping between $C^{1}$ Banach manifolds $M, N$ and let $S$ be a nonempty subset of $M$. If $v \in T_{x} M$ is a tangent vector to $S$ at $x \in S$, then the vector $D f_{x}(v)$ is tangent to $f(S)$ at the point $f(x)$. Moreover, if $f$ is a submersion at $x \in S$, then $v \in T_{x} M$ is tangent to $S$ at $x \in S$ if and only if $D f_{x}(v)$ is tangent to $f(S)$ at $f(x)$.

This notion of tangency to sets was introduced in connection with flow-invariance problems.

Definition 1.2 ([11]). A nonempty subset $S$ of a $C^{2}$ manifold $M$ is called flow-invariant with respect to a locally Lipschitz vector field $X$ on $M$ if every integral curve of $X$ starting from $S$ remains in $S$ as long as it exists.

The following characterization of flow-invariant sets extends the classical Nagumo-Brézis result.

TheOREM 1.3 ([11]). Let $S$ be a nonempty closed subset of a $C^{2}$ Banach manifold $M$ and let $X$ be a locally Lipschitz vector field on $M$. Then $S$ is flow-invariant with respect to $X$ if and only if the vector field $X$ is tangent to the set $S$.

For other flow-invariance results including a characterization of flowinvariant sets with respect to second order differential equations we refer to [12] and [13].

In order to use the tangent vectors to sets in constrained minimization problems we introduce the notion of critical point relative to a subset.

Definition 1.3. Let $S$ be a nonempty subset of $a C^{1}$ Banach manifold $M$ and let $f: M \rightarrow R$ be a differentiable function on $M$. A point $x \in S$ is called a critical point of the function $f$ relative to the subset $S$ (and then $f(x)$ is a critical value of $f$ relative to $S)$ if $D f_{x}(v)=0$ for all tangent vectors $v$ to $S$ at $x$.

We need the following characterization of tangent vectors. 
Lemma 1.4. A vector $v \in T_{x} M$ is tangent to the subset $S$ of a $C^{1}$ Banach manifold $M$ at $x \in S$ if and only if there exists a function $h \in(-\alpha, \alpha) \rightarrow u_{h}$ $\in T_{x} M$ (with $\alpha>0$ ) such that the following two conditions hold:

(i) $u_{h} \rightarrow 0$ in $T_{x} M$ as $h \rightarrow 0$;

(ii) if $\varphi: U \rightarrow E$ is any chart of $M$ at $x$ one has

$$
\varphi^{-1}\left(\varphi(x)+h D \varphi_{x}\left(v+u_{h}\right)\right) \in S
$$

for all $h \in(-\alpha, \alpha)$.

Proof. Assume that the vector $v \in T_{x} M$ is tangent to the set $S$ at $x \in S$ and let $\varphi: U \rightarrow E$ be a chart of $M$ at $x$. For each $h \neq 0$ there is a point $w_{h} \in \varphi(U \cap S)$ such that

$$
\left\|\varphi(x)+h D \varphi_{x}(v)-w_{h}\right\| \leqslant d\left(\varphi(x)+h D \varphi_{x}(v) ; \varphi(U \cap S)\right)+h^{2} .
$$

By Definition 1.1 and relation (1.1) we obtain

$$
\lim _{h \rightarrow 0} \frac{1}{h}\left\|\varphi(x)+h D \varphi_{x}(v)-w_{h}\right\|=0 .
$$

If we denote

$$
u_{h}=h^{-1} D \varphi_{x}^{-1}\left(w_{h}-\varphi(x)-h D \varphi_{x}(v)\right),
$$

then (1.2) implies (i) while (ii) is clearly satisfied.

Conversely, assume that for each $h \neq 0$ small enough there exists $u_{h} \in$ $T_{x} M$ satisfying (i) and (ii). It follows that

$$
w_{h}=\varphi(x)+h D \varphi_{x}\left(v+u_{h}\right) \in \varphi(U \cap S)
$$

and consequently

$$
\begin{aligned}
& \frac{1}{h} d\left(\varphi(x)+h D \varphi_{x}(v) ; \varphi(S \cap U)\right) \leqslant \frac{1}{|h|}\left\|\varphi(x)+h D \varphi_{x}(v)-w_{h}\right\| \\
& =\left\|D \varphi_{x}\left(u_{h}\right)\right\| \longrightarrow 0
\end{aligned}
$$

as $h \rightarrow 0$. Therefore the vector $v \in T_{x} M$ is tangent to $S$ at $x$.

Proposition 1.5. The set $T_{x} S$ of tangent vectors to the subset $S$ of a $C^{1}$ Banach manifold $M$ at $x \in S$ is a cone. If the set $S$ is locally convex (in the sense that at every point there is a chart $\varphi: U \rightarrow E$ of $M$ such that $\varphi(U \cap S)$ is a convex subset of $E)$, then $T_{x} S$ is a vector subspace of $T_{x} M$.

Proof. This follows immediately by applying Lemma 1.4.

From Lemma 1.4 we derive also the following principle of optimum 
THEOREM 1.6. Let $S$ be a nonempty subset of a $C^{1}$ Banach manifold $M$. If a differentiable function $f: M \rightarrow R$ has a minimum (maximum) over $S$ at $x \in S$, then $x$ is a critical point of $f$ relative to $S$.

Proof. Let us suppose that the function $f$ admits a minimum on $S$ at $x \in S$ and let $v \in T_{x} M$ be a tangent vector to $S$ at $x$. In view of Lemma 1.4, corresponding to each $h \in R$ sufficiently small (say $h \in(-\alpha, \alpha)$ ) there exists $u_{h} \in T_{x} M$ satisfying the above conditions (i) and (ii). By (ii) we have

$$
f \varphi^{-1}\left(\varphi(x)+h D \varphi_{x}\left(v+u_{h}\right)\right) \geqslant f(x) .
$$

Since $f$ is differentiable at $x$, it follows

$$
f_{\varphi^{-1}}\left(\varphi(x)+h D \varphi_{x}\left(v+u_{h}\right)\right)=f(x)+h D f_{x}\left(v+u_{h}\right)+o(h) .
$$

Then (1.3), (1.4) and condition (i) imply $D f_{x}(v)=0$ which ends the proof.

We state now a version for Condition (C) of R. Palais and S. Smale ([14], [15], [16]) in terms of our generalized tangency. Let $M$ be a Riemannian manifold, $S$ a nonempty subset of $M$ and $f: M \rightarrow R$ a differentiable function. For every $x \in S$ denote

(1.5) $\left\|D f_{x}\right\|_{S, x}=\sup \left\{\left|D f_{x}(v)\right| ; v\right.$ is a tangent vector to $S$ at $x$ and $\left.\|v\|_{x} \leqslant 1\right\}$.

Definition 1.4. A differentiable function $f: M \rightarrow R$ defined on a Riemannian manifold $M$ is said to satisfy Condition (C) relative to a subset $S$ of $M$ if whenever $\left\{x_{j}\right\}$ is a sequence in $S$ such that $f\left(x_{j}\right)$ is bounded and $\left\|D f_{x_{j}}\right\|_{S, x_{j}} \rightarrow 0$ as $j \rightarrow \infty$, then $\left\{x_{j}\right\}$ has a subsequence converging in $M$.

This constrained form of Condition (C) implies the following extension of Palais-Smale Existence Theorem ([14], [15], [16]).

THEOREM 1.7. Let $S$ be a closed subset of a complete Riemannian manifold $M$ and let $f: M \rightarrow R$ be a differentiable function. Assume that the function $f$ is bounded from below on $S$ and satisfies Condition (C) relative to the subset $S$. Then $\left.f\right|_{S}$ attains its greatest lower bound.

Proof. Apply the Ekeland's variational principle ([1], [2]) for $\left.f\right|_{s}$. Then for $\varepsilon>0$ there is a point $x_{\varepsilon} \in S$ such that

$$
f\left(x_{\varepsilon}\right) \leq \inf _{S} f+\varepsilon^{2}
$$

and

$$
f(x)>f\left(x_{\varepsilon}\right)-\varepsilon d\left(x, x_{\varepsilon}\right), \text { for all } x \in S, \quad x \neq x_{\varepsilon},
$$


where $d\left(x, x_{\varepsilon}\right)$ denotes the distance in $M$ between $x$ and $x_{\varepsilon}$ which is induced by the Riemannian structure. Take in (1.7) $x=\exp _{x_{\varepsilon}}(t v)$ with $t>0$ and $v$ an arbitrary tangent vector to $S$ at $x_{\varepsilon}$. Since, for $t$ sufficiently small,

$$
d\left(\exp _{x_{\varepsilon}}(t v), x_{\varepsilon}\right)=t\|v\|_{x_{\varepsilon}},
$$

one obtains

$$
f\left(\exp _{x_{\varepsilon}}(t v)\right)-f\left(x_{\varepsilon}\right)>-\varepsilon t\|v\|_{x_{s}}
$$

Letting $t \rightarrow 0$ it follows

$$
D f_{x_{\varepsilon}}(v) \geqslant-\varepsilon\|v\|_{x_{\varepsilon}},
$$

which, in view of (1.5), yields

$$
\left\|D f_{x_{\varepsilon}}\right\|_{S, x_{\varepsilon}} \leqslant \varepsilon .
$$

This allows one to apply Condition (C) and thus for $\varepsilon \rightarrow 0,\left\{x_{\varepsilon}\right\}$ converges (on a subsequence) to some point $z \in S$. From (1.6), by passing to limit, it results $f(z)=\inf _{S} f$ which completes the proof.

In the rest of this section we denote by $S$ a nonempty closed subset of a $C^{k}$ Riemannian manifold with $k \geqslant 2$. For every $x \in S$ let us denote by $T_{x} S$ the set of tangent vectors to $S$ at the point $x$. Admit now that $S$ satisfies the following regularity hypotheses:

$\left(\mathrm{H}_{1}\right) \quad T_{x} S$ is a closed vector subspace of $T_{x} M$, for all $x \in S$;

$\left(\mathrm{H}_{2}\right)$ The function $\operatorname{Pr}: T M \mid S \rightarrow T M$ mapping $v \in T_{x} M$

(with $x \in S$ ) to the orthogonal projection $\operatorname{Pr}(v)$ of $v$ on $T_{x} S$ can be extended to a locally Lipschitz map on a neighborhood of $T M \mid S=\cup_{x \in S} T_{x} M$ in $T M$.

Remark. Simple examples show that conditions $\left(\mathrm{H}_{1}\right),\left(\mathrm{H}_{2}\right)$ do not imply on $S$ to be a smooth submanifold of $M$. For instance, the set

$$
S=\left\{(x, y) \in R^{2} ; x^{2}+y^{2}-2 x=0 \text { or } x^{2}+y^{2}+2 x=0\right\}
$$

satisfies conditions $\left(\mathrm{H}_{1}\right),\left(\mathrm{H}_{2}\right)$ in $M=R^{2}$, but it is not a smooth submanifold of $R^{2}$. Proposition 1.5 shows that a sufficient condition for $T_{x} S$ to form a vector subspace of $T_{x} M$ is that the set $S$ be locally convex in $M$.

If $f: M \rightarrow R$ is a $C^{2}$ function on $M$, then, according to $\left(\mathrm{H}_{1}\right)$, the Riesz representation theorem ensures the existence of a unique tangent vector $\operatorname{grad}_{S} f(x) \in T_{x} S$ called the gradient of $f$ relative to $S$ at $x \in S$ and defined by 


$$
\left\langle\operatorname{grad}_{s} f(x), v\right\rangle_{x}=D f_{x}(v),
$$

for all $v \in T_{x} S$, where $\langle,\rangle_{x}$ denotes the scalar product on $T_{x} M$ determined by the Riemannian structure.

By hypothesis $\left(\mathrm{H}_{2}\right)$ it follows that $\operatorname{grad}_{S} f$ can be extended on a neighborhood of $S$ to a locally Lipschitz vector field. This is clear from the relation

$$
\operatorname{grad}_{s} f(x)=\operatorname{Pr}(\operatorname{grad} f(x)) \quad \text { with } x \in S .
$$

The following property of the integral curves of the vector field $\operatorname{grad}_{s} f$ generalizes a well-known result concerning the usual gradient field of a smooth real-valued function (see Palais [[14], § 10]).

Proposition 1.8. Let $S$ be a closed subset of a $C^{k}$ complete Riemannian manifold $(k \geqslant 2)$ such that the regularity conditions $\left(\mathrm{H}_{1}\right)$ and $\left(\mathrm{H}_{2}\right)$ hold. Let $f: M \rightarrow R$ be a $C^{2}$ function on $M$ which is bounded on $S$ and satisfies Condition (C) relative to the subset $S$. Then every maximum integral curve $\gamma$ of $\operatorname{grad}_{S} f$ with $x=\gamma(0) \in S$ is defined on $R$ and $\gamma(t)$ has critical points of $f$ relative to $S$ as limit points for $t \rightarrow \pm \infty$.

Proof. By hypotheses $\left(\mathrm{H}_{1}\right)$ and $\left(\mathrm{H}_{2}\right)$, $\operatorname{grad}_{s} f$ is a locally Lipschitz vector field which is tangent to the set $S$. Theorem 1.3 implies that

$$
\gamma(t) \in S \quad \text { for all } t \in\left(t^{-}(x), t^{+}(x)\right)=\operatorname{dom} \gamma .
$$

From (1.8) and (1.9) we deduce that the function $t \rightarrow f(\gamma(t))$ is bounded and monotone, so has finite limits at $t \rightarrow \pm \infty$. Prove for example that $t^{+}(x)$ $=\infty$. The same argument as in Palais [[14], Lemma p. 314] shows that

$$
\int_{0}^{t^{+}(x)}\left\|\operatorname{grad}_{S} f(\gamma(t))\right\|_{r(t)}^{2} d t<\infty
$$

so the length of $\gamma \mid\left[0, t^{+}(x)\right)$ is finite and therefore $\gamma\left(\left[0, t^{+}(x)\right)\right)$ is a relatively compact subset of $M$ (see Palais [[15]. Chapter IV B]). On the other hand if $t^{+}(x)<\infty, \gamma(t)$ cannot have limit points in $M$ as $t \rightarrow t^{+}(x)$ (cf. Palais [[14], §6] or [[15], Chapter VI A]). The contradiction shows $t^{+}(x)=\infty$. Then (1.10) implies that there exists a sequence $\left\{t_{n}\right\}$ of positive numbers such that $t_{n} \rightarrow \infty$ and $\left\|D f_{r\left(t_{n}\right)}\right\|_{S, r\left(t_{n}\right)} \rightarrow 0$ (see (1.5), (1.8)).

Furthermore, by (1.9), $f\left(\gamma\left(t_{n}\right)\right)$ is bounded. Hence one can apply Condition (C) to deduce that (on a subsequence) $r\left(t_{n}\right)$ converges in $M$ as $n \rightarrow \infty$ to a point $p \in S$. Passing to limit in

$$
\left\|\operatorname{grad}_{S} f\left(\gamma\left(t_{n}\right)\right)\right\|_{\gamma\left(t_{n}\right)}=\left\|D f_{\gamma\left(t_{n}\right)}\right\|_{s, \gamma\left(t_{n}\right)}
$$


we deduce from $\left(H_{2}\right)$ that $\operatorname{grad}_{S} f$ vanishes at $p$, so $p$ is a critical point of $f$ relative to $S$. The result is proved.

For a later use we state

Corollary 1.9. Let $M, S, f$ be as in Proposition 1.8. If $\left\{\gamma_{t}: S \rightarrow S\right\}_{t \in R}$ denotes the flow of the vector field $\left(-\operatorname{grad}_{S} f\right)$ on $S$, then for any compact subset $K$ of $M$ with $K \subset S$,

$$
C_{K}=\lim _{t \rightarrow \infty} \max _{x \in K} f\left(\gamma_{t}(x)\right)
$$

is a critical value of $f$ relative to the subset $S$.

Proof. The limit in (1.11) exists and is finite because the function $t \rightarrow \max _{x \in K} f\left(\gamma_{t}(x)\right)$ is continuous, decreasing and bounded. Thus for any positive integer $n$ there are $\delta_{n}>0$ and a point $x_{n} \in K$ such that

$$
\left|f\left(\gamma_{t}\left(x_{n}\right)\right)-C_{K}\right|<\frac{1}{n}, \quad \text { if } t \geqslant \delta_{n} .
$$

Letting $t \rightarrow \infty$ in the above inequality, Proposition 1.8 implies that there exists a sequence $\left\{y_{n}\right\}$ of critical points of $f$ relative to $S$ such that

$$
\left|f\left(y_{n}\right)-C_{K}\right|<\frac{1}{n}, \quad \text { for every } n .
$$

The generalized condition (C) given in Definition 1.4 insures that (on a subsequence) $\left\{y_{n}\right\}$ converges to a point $z \in S$. Arguing as in the final part of the proof of Theorem 1.7 we derive that $z$ is a critical point of $f$ relative to $S$. By (1.12) we get $f(z)=C_{K}$. The proof is complete.

\section{§2. Geodesics as critical points relative to sets}

Throughout this section $M$ denotes a smooth Riemannian manifold, possibly infinite dimensional, with the Riemannian structure $\langle,\rangle_{x}$. Consider the Riemannian manifold $L_{1}^{2}(I ; M)$ of absolutely continuous maps from the unit interval $I=[0,1]$ to $M$ with locally square integrable derivative. The tangent space $T_{c} L_{1}^{2}(I, M)$ at $c \in L_{1}^{2}(I ; M)$ consists of all absolutely continuous vector fields $X$ along $c$ with square integrable covariant derivative $\nabla_{c} X$. The Riemannian structure of $L_{1}^{2}(I ; M)$ is given by

$$
\langle X, Y\rangle_{c}=\int_{0}^{1}\left(\langle X(t), Y(t)\rangle_{c(t)}+\left\langle\nabla_{c} X(t), \nabla_{c} Y(t)\right\rangle_{c(t)}\right) d t
$$

where $X$ and $Y$ are arbitrary elements of $T_{c} L_{1}^{2}(I ; M)$. 
If $N$ is a closed subset of $M \times M$, denote

$$
\Lambda_{N}(M)=\left\{c \in L_{1}^{2}(I ; M) ;(c(0), c(1)) \in N\right\} .
$$

The tangent vectors to $A_{N}(M)$ (in the sense of Definition 1.1) are characterized as follows.

Lemma 2.1. A vector $X \in T_{c} L_{1}^{2}(I ; M)$ is tangent to the set $\Lambda_{N}(M)$ at $c \in \Lambda_{N}(M)$ if and only if $(X(0), X(1))$ is a tangent vector to the set $N$ at the point $(c(0), c(1)) \in N$.

Proof. Since the map from $L_{1}^{2}(I ; M)$ to $M \times M$ given by $\omega \rightarrow(\omega(0)$, $\omega(1))$ is a submersion, we have only to apply the second part of Theorem 1.2 .

The energy integral $E: L_{1}^{2}(I ; M) \rightarrow R$ is defined by

$$
E(c)=\frac{1}{2} \int_{0}^{1}\left\|c^{\prime}(t)\right\|_{c(t)}^{2} d t .
$$

Recall that the function $E$ is smooth and the differential $D E_{c}$ of $E$ at $c \in L_{1}^{2}(I ; M)$ is the following

$$
D E_{c}(X)=\int_{0}^{1}\left\langle\nabla_{c} X(t), c^{\prime}(t)\right\rangle_{c(t)} d t \quad \text { for all } X \in T_{c} L_{1}^{2}(I ; M) .
$$

We refer to [5], [9] and [18] for the general study of the energy functional in connection with the theory of geodesics.

The following result is an extension of Theorem 1.6 (1) in Grove [4] and deals with the sets $\Lambda_{N}(M)$ (see (2.2)) when $N=S_{0} \times S_{1}$.

Theorem 2.2. Let $S_{0}$ and $S_{1}$ be two nonempty closed subsets of the Riemannian manifold $M$. Then $c \in \Lambda_{S_{0} \times S_{1}}(M)$ is a critical point of $E$ relative to $\Lambda_{S_{0} \times S_{1}}(M)$ if and only if $c$ is a geodesic of $M$ from $S_{0}$ to $S_{1}$ which is orthogonal to $S_{i}, i=0,1$, i.e. $c^{\prime}(0) \in\left(T_{c(0)} S_{0}\right)^{\perp}$ and $c^{\prime}(1) \in\left(T_{c(1)} S_{1}\right)^{\perp}$, where by $T_{x} S_{i}$ we denoted the set of tangent vectors to $S_{i}$ at a point $x$.

Proof. Assume that $c \in \Lambda_{S_{0} \times S_{1}}(M)$ is a critical point of $E$ relative to the subset $\Lambda_{S_{0} \times S_{1}}(M)$ of $L_{1}^{2}(I ; M)$, that is

$$
D E_{c}(X)=0 \quad \text { for all tangent vectors } X \text { to } \Lambda_{S_{0} \times S_{1}}(M) \text { at } c \text {. }
$$

If $p=c(0) \in S_{0}$ and $q=c(1) \in S_{1}$ are the endpoints of the curve $c$, then $c$ belongs to the smooth submanifold

$$
\Lambda_{p, q}(M)=\left\{\omega \in L_{1}^{2}(I ; M) ; \omega(0)=p, \omega(1)=q\right\}
$$


of the manifold $L_{1}^{2}(I ; M)$. By Lemma 2.1 it is clear that the tangent space

$$
T_{c} \Lambda_{p, q}(M)=\left\{X \in T_{c} L_{1}^{2}(I ; M) ; X(0)=0, X(1)=0\right\}
$$

is contained in the set of tangent vectors to $\Lambda_{S_{0} \times S_{1}}(M)$ at $c$, so (2.4) implies

$$
D E_{c}(X)=0 \quad \text { for all } X \in T_{c} \Lambda_{p, q}(M) .
$$

Then it follows that $c$ is a geodesic of $M$ (see, e.g., Grove [[4], Proposition 1.5] or Schwartz [[18], Theorem 6.30]). This fact combined with (2.3) and (2.4) yields

$$
D E_{c}(X)=\left\langle X(1), c^{\prime}(1)\right\rangle_{c(1)}-\left\langle X(0), c^{\prime}(0)\right\rangle_{c(0)}=0
$$

for all tangent vectors $X$ to $\Lambda_{S_{0} \times S_{1}}(M)$ at $c$. In view of Lemma 2.1, we deduce from (2.5) that for all $v_{0} \in T_{c(0)} S_{0}$ and $v_{1} \in T_{c(1)} S_{1}$ holds

$$
\left\langle v_{0}, c^{\prime}(0)\right\rangle_{c(0)}=\left\langle v_{1}, c^{\prime}(1)\right\rangle_{c(1)}
$$

which clearly implies $c^{\prime}(0) \in\left(T_{c(0)} S_{0}\right)^{\perp}$ and $c^{\prime}(1) \in\left(T_{c(1)} S_{1}\right)^{\perp}$.

Conversely, assume that $c \in \Lambda_{S_{0} \times S_{1}}(M)$ is a geodesic on $M$ with $c^{\prime}(0) €$ $\left(T_{c(0)} S_{0}\right)^{\perp}$ and $c^{\prime}(1) \in\left(T_{c(1)} S_{1}\right)^{\perp}$. Since $c$ is a geodesic, one can write (2.3) in the form

$$
D E_{c}(X)=\left\langle X(1), c^{\prime}(1)\right\rangle_{c(1)}-\left\langle X(0), c^{\prime}(0)\right\rangle_{c(0)},
$$

for all $X \in T_{c} L_{1}^{2}(I ; M)$. Taking into account Lemma 2.1, the orthogonality hypothesis and (2.6), it results that $D E_{\mathrm{c}}(X)=0$ for each $X$ belonging to the set of tangent vectors to $\Lambda_{S_{0} \times S_{1}}(M)$ at $c$. This completes the proof.

Remark. If the sets $S_{0}$ and $S_{1}$ are smooth submanifolds of $M$, then Theorem 2.2 reduces to Grove's Theorem 1.6 (1) from [4].

Corollary 2.3. A minimizing geodesic between two closed subset $S_{0}$ and $S_{1}$ of $M$ is orthogonal to $S_{0}$ and $S_{1}$. Moreover, if in a homotopy class of curves joining $S_{0}$ and $S_{1}$ a geodesic $c$ has minimal length, then $c$ is orthogonal to $S_{0}$ and $S_{1}$.

Proof. It is sufficient to prove the first statement because the second part follows by arguing on the component of $c$. Thus let $c$ be a minimizing geodesic from $S_{0}$ to $S_{1}$, that is the length of $c$ equals

$$
d\left(S_{0}, S_{1}\right)=\inf _{x_{i} \in S_{i}, i=0,1} d\left(x_{0}, x_{1}\right) .
$$

It results that $c$ minimizes the energy integral $E$ over the subset $\Lambda_{S_{0} \times S_{1}}(M)$ of $L_{1}^{2}(I ; M)$ (see, e.g., Milnor [[9], Lemma 12.1]). We apply Theorem 1.6 
to deduce that $c$ is a critical point for $E$ relative to the set $\Lambda_{S_{0} \times S_{1}}(M)$ (in the sense of Definition 1.3). The result follows now from Theorem 2.2.

The above result yields the following generalization of a classical result [[14], Theorem 2.6] from the case of closed submanifolds to arbitrary closed subsets.

Corollary 2.4. Let $M$ be a finite dimensional complete and connected Riemannian manifold. Let $S_{0}$ and $S_{1}$ be two closed subsets of $M$ provided at least one of them is compact. Then there exists a geodesic starting orthogonally from $S_{0}$ and ending orthogonally to $S_{1}$ with length equal to $d\left(S_{0}, S_{1}\right)$. Moreover in any homotopy class of curves joining $S_{0}$ and $S_{1}$ there is a geodesic of minimal length and orthogonal to $S_{i}, i=0,1$.

Proof. A simple argument based on the Hopf-Rinow Theorem shows that there exists a minimizing geodesic between $S_{0}$ and $S_{1}$ (see the proof of Theorem 1 in [10]). Hence it suffices to apply Corollary 2.3. The second part follows analogously.

Remark. It is well-known that on infinite dimensional Riemannian manifolds the Hopf-Rinow Theorem is generally not true (see Ekeland [2] for a counterexample). Therefore in Corollary 2.4 we cannot drop the hypothesis on $M$ to be finite dimensional.

As a further application of tangent vectors to sets we shall extend a general result due to Kurogi ([6], [7]) concerning the geodesics with the same angle.

Let $f$ be an isometry of the Riemannian manifold $M$. If we denote the graph of $f$ by $G(f)$, then

$$
\Lambda_{G(f)}(M)=\left\{\omega \in L_{1}^{2}(I ; M) ; f(\omega(0))=\omega(1)\right\}
$$

is a smooth submanifold of $L_{1}^{2}(I ; M)$ (see Grove [4]). If $S$ is a nonempty subset of $M$, denote

$$
\Lambda(S, f)=\Lambda_{G(f) \cap(S \times M)}(M)=\left\{\omega \in \Lambda_{G(f)}(M) ; \omega(0) \in S\right\} .
$$

Lemma 2.5. If $c \in \Lambda(S, f)$, then $X \in T_{c} L_{1}^{2}(I ; M)$ is tangent to $\Lambda(S, f)$ if and only if $D f_{c(0)}(X(0))=X(1)$ (i.e. $X \in T_{c} \Lambda_{G(f)}(M)$ ) and the vector $X(0) \in$ $T_{c(0)} M$ is tangent to $S$ at $c(0)$.

Proof. The result follows from Lemma 2.1 for the case $N=G(f) \cap$ $(S \times M)$ and the fact that $G(f)$ is a smooth submanifold of $M \times M$. 
The consideration of the set $\Lambda(S, f)$ given by (2.7) is motivated by its closed relation with the problem of geodesics acrossing the set $S$ with the same angle.

Definition 2.1. Let $S$ be a nonempty subset of a finite dimensional Riemannian manifold $M$. A geodesic $c: I \rightarrow M$ with $(c(0),(c(1)) \in S \times S$ forms the same angle with the set $S$ if

$$
\Varangle\left(c^{\prime}(0), \operatorname{Span} T_{c(0)} S\right)=\Varangle\left(c^{\prime}(1), \operatorname{Span} T_{c(1)} S\right),
$$

where Span $T_{c(0)} S$ and Span $T_{c(1)} S$ denote the vector subspaces of $T_{c(0)} M$ and $T_{c(1)} M$ spanned by the sets of tangent vectors $T_{c(0)} S$ and $T_{c(1)} S$ respectively.

In the case when $S$ is just a smooth submanifold of $M$, Definition 2.1 reduces to Definition 2.3 of Kurogi [7] (see also Kurogi [6] for the case of submanifolds of codimension 1 ).

Theorem 2.6. Let $S$ be a closed subset of a finite dimensional Riemannian manifold $M$ and let $f$ be an isometry of $M$ such that $f(S)=S$. Then $c \in \Lambda(S, f)$ is a critical point of the energy integral $E$ relative to the subset $\Lambda(S, f)$ of $L_{1}^{2}(I ; M)$ if and only if $c$ is a geodesic on $M$ such that $c^{\prime}(1)-$ $D f_{c(0)}\left(c^{\prime}(0)\right)$ is orthogonal to $T_{c(1)} S$. Moreover, if $c$ is a critical point of $E$ relative to $\Lambda(S, f)$, then $c$ is a geodesic on $M$ with the same angle for the set $S$.

Proof. Assume that $c$ is a critical point for $E$ relative to the subset $\Lambda(S, f)$ of $L_{1}^{2}(I ; M)$. Then

$$
D E_{c}(X)=0 \text { for each tangent vector } X \text { to } \Lambda(S, f) \text { at } c \text {. }
$$

Arguing as in the proof of Theorem 2.2, relations (2.3) and (2.9) imply that $c$ is a geodesic on $M$ and furthermore

$$
D E_{c}(X)=\left\langle X(1), c^{\prime}(1)\right\rangle_{c(1)}-\left\langle X(0), c^{\prime}(0)\right\rangle_{c(0)}=0
$$

for all tangent vectors $X$ to $\Lambda(S, f)$ at $c$. Taking into account that $f$ is an isometry of $M$ and using Lemma 2.5 we deduce from (2.10) the equality

$$
\left\langle X(1), c^{\prime}(1)-D f_{c(0)}\left(c^{\prime}(0)\right)\right\rangle_{c(1)}=0,
$$

if $X$ belongs to the set of tangent vectors to $\Lambda(S, f)$ at $c$. Notice that when $X$ is an arbitrary tangent vector to $\Lambda(S, f)$ at $c, X(1)$ is an arbitrary tangent vector to $S$ at $c(1)$ (see Theorem 1.2). Consequently (2.11) implies 
that $c^{\prime}(1)-D f_{c(0)}\left(c^{\prime}(0)\right)$ is orthogonal to $T_{c(1)} S_{1}$.

Conversely, suppose that $c$ is a geodesic on $M$ such that

$$
c^{\prime}(1)-D f_{c(0)}\left(c^{\prime}(0)\right) \in\left(T_{c(1)} S_{1}\right)^{\perp} .
$$

This yields (2.11) which, under our assumptions, clearly insures (2.9). Therefore we proved the sufficiency part.

To check the last assertion of Theorem 2.7 assume now again that $c \in \Lambda(S, f)$ is a critical point of $E$ relative to $\Lambda(S, f)$. Then, according to the necessity part of the proof, $c^{\prime}(1)-D f_{c(0)}\left(c^{\prime}(0)\right)$ is orthogonal to $T_{c(1)} S_{1}$ and thus

$$
\left\langle c^{\prime}(1)-D f_{c(0)}\left(c^{\prime}(0)\right), D f_{c(0)} P_{0}\left(c^{\prime}(0)\right)\right\rangle_{c(1)}=0,
$$

where $P_{0}\left(c^{\prime}(0)\right)$ denotes the orthogonal projection of $c^{\prime}(0)$ on Span $T_{c(0)} S$. Since $f$ is an isometry, from (2.12) we deduce

$$
\left\langle c^{\prime}(0), P_{0}\left(c^{\prime}(0)\right)\right\rangle_{c(0)}=\left\langle c^{\prime}(1), P_{1}\left(c^{\prime}(1)\right)\right\rangle_{c(1)},
$$

where $P_{1}\left(c^{\prime}(1)\right)$ represents the orthogonal projection of $c^{\prime}(1)$ on Span $T_{c(1)} S$. Therefore (2.8) holds which concludes the proof.

Remark. If the set $S$ is a smooth submanifold of $M$, we obtain from the last part of Theorem 2.6 the result of Kurogi [[7], Theorem 4] (see also [[6], Theorem 1.3] for submanifolds of codimension 1).

We end this section with an extension of Theorem 2.4 in Grove [4] concerning Condition (C) for the energy integral on $\Lambda_{N}(M)$. Our result provides a basic example of functional satisfying Condition (C) relative to a set in the sense of Definition 1.4.

Theorem 2.7. Let $M$ be a finite dimensional complete Riemannian manifold and let $N$ be a closed subset of $M \times M$ such that at least one of the projections $\operatorname{pr}_{i}(N), i=1,2$, is a compact subset of $M$. Then the energy integral $E: L_{1}^{2}(I ; M) \rightarrow R$ satisfies Condition (C) relative to the subset $\Lambda_{N}(M)$ of $L_{1}^{2}(I ; M)$.

Proof. We follow the same lines as in the proof of Grove [[4], Theorem 2.4], Let $\left\{c_{j}\right\}$ be a sequence in $\Lambda_{N}(M)$ such that $E\left(c_{j}\right)$ is bounded and $\left\|D E_{c_{j}}\right\|_{\Lambda_{N}(M), c_{j}} \rightarrow 0$ as $j \rightarrow \infty$. The same argument as in [4] shows that there exist constants $\lambda>0$ and $C$ such that

$$
\lambda\left\|X_{i}-X_{j}\right\| \leqslant C\left\|X_{i}-X_{j}\right\|_{\infty}^{2}+\left(D\left(E \circ \exp _{a}\right)_{X_{i}}-D\left(E \circ \exp _{a}\right)_{X_{j}}\right)\left(X_{i}-X_{j}\right),
$$


where we denoted by $\exp _{a}: T_{a} L_{1}^{2}(I ; M) \rightarrow L_{1}^{2}(I ; M)$ the natural exponential chart on $L_{1}^{2}(I ; M)$ at some $a \in C^{\infty}(I ; M), X_{j}=\exp _{a}{ }^{-1}\left(c_{j}\right)$ for every $j,\|\|$ the norm on $T_{a} L_{1}^{2}(I ; M)$ induced by the Riemannian structure in (2.1) and \|\|$_{\infty}$ the uniform norm on $T_{a} L_{1}^{2}(I ; M)$. On the other hand, using the Schwartz inequality, one obtains from (2.3) and (2.1) the following estimate

$$
\left\|D E_{c_{j}}\right\|_{c_{j}} \leqslant\left(2 E\left(c_{j}\right)\right)^{1 / 2} \text {. }
$$

The same argument as in [4] shows that there exists a decomposition

$$
X_{j}=X_{j}^{0}+Y_{j} \quad \text { for all } j
$$

such that $D\left(\exp _{a}\right)_{X_{j}}\left(X_{j}^{0}\right)$ is a tangent vector to $\Lambda_{N}(M)$ at $c_{j}$ for each $j$, while $Y_{j}$ belongs to a finite dimensional vector space $V$ independent of $j$. Using the assumptions on the sequence $\left\{c_{j}\right\}$ together with (2.14), (2.15) and the boundedness of $\left\|X_{i}-X_{j}\right\|$, it follows from (2.13) that (on a subsequence) $\left\|X_{i}-X_{j}\right\| \rightarrow 0$ as $i, j \rightarrow \infty$. This proves the result.

\section{$\S 3$. Geodesics with the same angle for a compact set}

The aim of this section is to extend the existence result of T. Kurogi ([6], [7]) concerning geodesics acrossing a compact submanifold $S$ with the same angle to the general case of an arbitrary compact subset $S$. Namely we shall provide sufficient conditions insuring existence of nontrivial geodesics with the same angle for a given compact set $S$. Our approach is based on the theory of tangent vectors that we developed in Section 1.

Throughout this section $M$ denotes a complete and connected finite dimensional Riemannian manifold, $S$ a nonempty compact subset of $M$ and $f: M \rightarrow M$ an isometry of $M$ such that $f(S)=S$. We look for nontrivial geodesics $c: I \rightarrow M$ such that

$$
\begin{gathered}
c(0) \in S, \quad f(c(0))=c(1) \\
\Varangle\left(c^{\prime}(0), \operatorname{Span} T_{c(0)} S\right)=\Varangle\left(c^{\prime}(1), \operatorname{Span} T_{c(1)} S\right)
\end{gathered}
$$

(i.e. geodesics with the same angle for $S$ as introduced in Definition 2.1).

The first result in this direction is the following

THEOREM 3.1. If the compact set $S$ does not contain fixed points of the isometry $f$, then there exists a nontrivial geodesic satisfying (3.1) and (3.2).

Proof. According to the last part of Theorem 2.6 we need only to prove that there exists a critical point $c$ of the energy functional $E$ relative 
to the set $A(S, f)$ so that the curve $c$ is nontrivial. Since the set $S$ is supposed to be compact, by Theorem 2.7 it follows that the function $E$ satisfies Condition (C) relative to the subset $\Lambda(S, f)$ of $L_{1}^{2}(I ; M)$. Then Theorems 1.6 and 1.7 insure the existence of a critical point $c$ of $E$ relative to the set $\Lambda(S, f)$. The curve $c$ is necessarily nontrivial because $c \in \Lambda(S, f)$ and the isometry $f$ is without fixed points in $S$. This completes the proof.

Remark. If $S$ is a smooth submanifold of $M$, Theorem 3.1 reduces to a result of T. Kurogi ([7], [6]).

Identifying a constant curve with its single-element image, we have

$$
E^{-1}(0) \cap \Lambda(S, f)=\operatorname{Fix}(f) \cap S,
$$

where Fix $(f)$ denotes the fixed points of the mapping $f$.

Proposition 3.2. There exists some $\varepsilon>0$ such that, under the identification (3.3), Fix $(f) \cap S$ is a strong deformation retract of $E^{-1}[0, \varepsilon] \cap(S, f)$.

Proof. In order to use Generalized Morse Lemma (see Meyer [8]) consider a compact smooth submanifold $W$ of $M$ such that $S \subset \operatorname{Int} W$. The existence of $W$ is proved for example in Fuks and Rohlin ([3], p. 227). Then $\Lambda(W, f)$ is a smooth submanifold of $L_{1}^{2}(I ; M)$. Observe that corresponding to each component $F_{i}$ of Fix $(f) \cap W$ one can apply Generalized Morse Lemma [8] for the restriction of $E$ ० exp to a tubular neighborhood $V_{i}$ of $F_{i}$. Then, by the same argument as in [6], we deduce that there exist a diffeomorphism $\varphi_{W}$ near the 0 -section of $V$, an orthogonal projection $P_{W}$ and some constant $\delta_{W}>0$ such that

$$
E \circ \varphi_{W}(X)=\left\|P_{W}(X)\right\|^{2} \quad \text { for }\|X\|<\delta_{W},
$$

where $\|$ \| denotes the norm induced by the Riemannian structure of $\Lambda(W, f)$.

As in the proof of Corollary 3.3 in [4] and using essentially Condition (C), it follows from (3.4) that

$$
\varphi_{W}\left(\cup V_{i}\right)=(E \mid \Lambda(W, f))^{-1}\left[0, \varepsilon_{W}\right],
$$

with $\varepsilon_{W}=\delta_{W}^{2}$ and $V_{i}$ sufficiently small. Moreover, $\varphi\left(0_{x}\right)=x$, for every $x \in \operatorname{Fix}(f) \cap W$.

By (3.5) we see that $\operatorname{Fix}(f) \cap W$ is a strong deformation retract of $(E \mid \Lambda(W, f))^{-1}\left[0, \varepsilon_{W}\right]$ and let $G_{W}$ be the corresponding homotopy.

Choose now a sequence $\left\{W_{n}\right\}$ of compact smooth submanifolds of $M$ such that 


$$
\bigcap_{n} W_{n}=S \text { and } S \subset \operatorname{int} W_{n+1} \subset W_{n+1} \subset \operatorname{int} W_{n}
$$

for every $n$ (see [[3], p. 227]).

In view of (3.6), from the above construction one can see that, for each $n, \varphi_{W_{n+1}}$ is the restriction of $\varphi_{W_{n}}$ and $\varepsilon_{W_{n}} \geqslant \varepsilon>0$. Therefore, by passing to limit, the sequence of homotopies $\left\{G_{W_{n}}\right\}$ defines a homotopy $G$ : $(E \mid$ $\Lambda(S, f))^{-1}[0, \varepsilon] \times I \rightarrow(E \mid \Lambda(S, f))^{-1}[0, \varepsilon]$. Clearly $G$ inherits the properties of the homotopies $G_{W_{n}}$, so Fix $(f) \cap S$ is a strong deformation retract of $E^{-1}[0, \varepsilon]$ $\cap \Lambda(S, f)$ which proves the result.

The below lemma is the main step in generalizing the Kurogi's result from compact submanifolds to compact subsets. The proof relies on the concept of gradient field of a smooth real valued function relative to a set (in our case $\operatorname{grad}_{\Lambda(S, f)} E$ ) which we introduced in Section 1.

For our purpose it is now convenient to take the gradient of $E$ relative to $\Lambda(S, f)$ with respect to the Riemannian structure on $L_{1}^{2}(I ; M)$ used in Palais [14] or Schwartz [[18], Chapter 6], namely

$$
\langle X, Y\rangle_{c}=\langle X(0), Y(0)\rangle_{c(0)}+\int_{0}^{1}\left\langle X^{\prime}(t), Y^{\prime}(t)\right\rangle_{R^{n}} d t
$$

for all $X, Y \in T_{c} L_{1}^{2}(I ; M)$, where $M$ is isometrically embedded in an Euclidean space $R^{n}$. The advantage of this metric lies in the fact that, contrasting with (2.1), $\Lambda(S, f)$ endowed with the metric induced by (3.7) is locally isometric to the product of $S$ and a Hilbert space (see [14] or [18]).

Lemma 3.3. Let $f: M \rightarrow M$ be an isometry of the finite dimensional complete Riemannian manifold $M$ and $S$ a compact subset of $M$ satisfying condition $\left(\mathrm{H}_{1}\right),\left(\mathrm{H}_{2}\right)$ from Section 1 . If there are no nontrivial geodesics with the same angle for $S$, then under the identification (3.3) the inclusion $i$ : Fix $(f) \cap S \rightarrow \Lambda(S, f)$ is a weak homotopy equivalence.

Proof. If $L_{1}^{2}(I ; M)$ is endowed with Riemannian structure (3.7), the energy function $E: L_{1}^{2}(I ; M) \rightarrow R$ admits a locally Lipschitz gradient field $\operatorname{grad}_{\Lambda(S, f)} E$ relative to the set $\Lambda(S, f)$ in the sense of Section 1. Indeed, from $\left(\mathrm{H}_{1}\right)$, Lemma 2.5 and relation (3.7) it follows that for every $c \in \Lambda(S, f)$ the set of tangent vectors to $\Lambda(S, f)$ at $c$ forms a closed vector subspace of $T_{c} L_{1}^{2}(I ; M)$. Furthermore, $\Lambda(S, f)$ is locally the product of $S$ by a Hilbert space and the identification of the corresponding tangent spaces is an isometry under the metric (3.7). Therefore condition $\left(\mathrm{H}_{2}\right)$ for $S$ in 
$M$ implies condition $\left(\mathrm{H}_{2}\right)$ for $\Lambda(S, f)$ in $L_{1}^{2}(I ; M)$, so $\operatorname{grad}_{\Lambda(S, f)} E$ exists and is locally Lipschitz.

Turn now to the proof that for any integer $q \geqslant 0$, the induced homomorphism $i_{q}: \pi_{q}(\operatorname{Fix}(f) \cap S) \rightarrow \pi_{q}(\Lambda(S, f))$ is an isomorphism. We proceed as in Grove [[4], Lemma 3.4]. Let $i_{q}[g]=0$ and let $G: S_{q} \times I \rightarrow \Lambda(S, f)$ be the null-homotopy of $g$ in $\Lambda(S, f)$. Then Corollary 1.9 insures that

$$
C_{G}=\lim _{t \rightarrow \infty} \max _{c \in G\left(S^{q \times I)}\right.} E\left(\gamma_{t}(c)\right)
$$

is a critical value of $E$ relative to $\Lambda(S, f)$, where $\gamma_{t}$ denotes the flow on $\Lambda(S, f)$ defined by $\left(-\operatorname{grad}_{\Lambda(S, f)} E\right)$. Notice that Theorem 2.7 holds also when $L_{1}^{2}(I ; M)$ is endowed with the Riemannian structure (3.7). According to the assumption that there is no nontrivial geodesic with the same angle for $S$, the second part of Theorem 2.6 implies $C_{G}=0$. From (3.8) it follows that for $\varepsilon>0$ chosen as in Proposition 3.2 and $t$ sufficiently large,

$$
\gamma_{t}\left(G\left(S^{q} \times I\right)\right) \subset E^{-1}[0, \varepsilon] \cap \Lambda(S, f) .
$$

Then Proposition 3.2 shows that $g$ is null-homotopic in $\operatorname{Fix}(f) \cap S$, so $i_{q}$ is injective.

Now let $[h] \in \pi_{q}(\Lambda(S, f))$. Arguing as above, we derive

$$
\lim _{t \rightarrow \infty} \max _{c \in h(S q)} E\left(\gamma_{t}(c)\right)=0 \text {. }
$$

Hence for some $\varepsilon>0$ given by Proposition 3.2 and $t>0$ large enough we have

$$
\gamma_{t}\left(h\left(S^{q}\right)\right) \subset E^{-1}[0, \varepsilon] \cap \Lambda(S, f) .
$$

If $r: E^{-1}[0, \varepsilon] \cap \Lambda(S, f) \rightarrow \operatorname{Fix}(f) \cap S$ is the deformation retract from Proposition 3.2, then (3.9) yields $i_{q}\left[r \circ \gamma_{t} \circ h\right]=[h]$, so $i_{q}$ is also surjective. This concludes the proof.

We are now prepared to prove the main result of this section which together with Theorem 3.1 extends the Kurogi's existence theorem ([6], [7]).

THEOREM 3.4. Let $M$ be a finite dimensional complete Riemannian manifold, $S$ a pathwise connected compact subset of $M$ and $f$ an isometry of $M$ with $f(S)=S$. Suppose moreover that $M$ is simply connected, S satisfies conditions $\left(\mathrm{H}_{1}\right),\left(\mathrm{H}_{2}\right)$ and Fix $(f) \cap S$ is not pathwise connected. Then there exists in $M$ a nontrivial geodesic with the same angle for the set $S$.

Proof. Choose some point $p$ belonging to $\operatorname{Fix}(f) \cap S$ as the base point 
in $M$ and also in $\Lambda(S, f)$. If $G(f)$ denotes the graph of $f$, then we have the canonical fibration $P: \Lambda_{G(f)}(M) \rightarrow G(f) \equiv M$ given by $P(\omega)=(\omega(0), \omega(1))$. The restriction of the fibration $P$ to the subset $S$ of $M$ defines a fibration $\Lambda(S, f) \rightarrow S$ over $S$ whose fibre is $\Lambda_{p}(M)=\left\{\omega \in L_{1}^{2}(I ; M) ; \omega(0)=p\right\}$. The exactness of the homotopy sequence

$$
\cdots \longrightarrow \pi_{0}\left(\Lambda_{p}(M)\right) \longrightarrow \pi_{0}(\Lambda(S, f)) \longrightarrow \pi_{0}(S)
$$

of this fibration (see for example [[19], Chapter 7]) shows that under the required hypotheses on $M$ and $S$ we have $\pi_{0}(\Lambda(S, f))=0$. This implies the existence of a nontrivial geodesic with the same angle for the set $S$ because otherwise we arrive at a contradiction with Lemma 3.3. The result is established.

Remark. In particular, if the isometry $f$ of $M$ has only finitely many fixed points in $S$ but not exactly one Theorem 3.4 applies. Therefore, Theorems 3,1 and 3.4 extend completely the Kurogi's existence results ([6], [7]) on geodesics with the same angle for compact submanifolds to geodesics with the same angle for compact sets satisfying the natural regularity hypotheses $\left(\mathrm{H}_{1}\right),\left(\mathrm{H}_{2}\right)$.

\section{REFERENCES}

[ 1 ] I. Ekeland, On the variational principle, J. Math. Anal. Appl., 47 (1974), 324-353.

[2] - Nonconvex minimization problems, Bull. Amer. Math. Soc. (New Series), 1 (1979), 443-474.

[ 3 ] D. B. Fuks and V. A. Rohlin, Beginer's Course in Topology (Geometric chapters), Universitext, Springer-Verlag, Berlin, 1984.

[4] K. Grove, Condition (C) for the energy integral on certain path spaces and applications to the theory of geodesics, J. Differential Geom., 8 (1973), 207-223.

[5] W. Klingenberg, Lectures on closed geodesics, Grundlagen der mathematischen Wissenschaften, 230, Springer-Verlag, Berlin, 1978.

[6] T. Kurogi, On some types of geodesics on Riemannian manifolds, Nagoya Math. J., 81 (1981), 27-43.

[ 7 ] - On geodesics with the same angle, Proc. Japan Acad., 59 (1983), 427-430.

[ 8 ] W. Meyer, Kritische Mannigfaltigkeiten in Hilbertmannigfaltigkeiten, Math. Ann., 170 (1967), 45-66.

[ 9 ] J. Milnor, Morse theory, Ann. Math. Stud. 51, Princeton Univ. Press, Princeton, 1963.

[10] D. Motreanu, Optimization problems on complete Riemannian manifolds, Colloq. Math., to appear.

[11] D. Motreanu and N. H. Pavel, Quasi-tangent vectors in flow-invariance and optimization problems on Banach manifolds, J. Math. Anal. Appl., 88 (1982), 116-132.

[12] — Flot-invariance par rapport aux équations différentielles du second ordre sur une variété, C. R. Acad. Sc. Paris, 297 (1983), 157-160. 
[13] - Flow-invariance for second order differential equations on manifolds and orbital motions, Preprint Series in Math., 1 (1983), Univ. Iaşi.

[14] R. S. Palais, Morse theory on Hilbert manifolds, Topology, 2 (1963), 299-340.

[15] _ L Lectures on the differential topology on infinite-dimensional manifolds, Mimeographed notes, Brandeis Univ., 1964-1965.

[16] R. S. Palais and S. Smale, A generalized Morse theory, Bull. Amer. Math. Soc., 70 (1964), 165-172.

[17] N. H. Pavel, Differential equations, flow invariance and applications, Research Notes in Math., 113, Pitman, London, 1984.

[18] J. Schwartz, Nonlinear functional analysis, Gordon and Breach, New York, 1969.

[19] E. Spanier, Algebraic Topology, McGraw-Hill, New York, 1966.

Department of Mathematics

University of Iasi

6600 Iasi, Romania 